\title{
Hybrid metal-dielectric metasurfaces for refractive index sensing Supporting information
}

Debdatta Ray, ${ }^{\dagger}$ T.V. Raziman, ${ }^{\dagger}$ Christian Santschi, ${ }^{\dagger}$ Dordaneh Etezadi, ${ }^{\ddagger}, \delta$ Hatice Altug,,+ and Olivier J.F. Martin ${ }^{*, \dagger}$

$\dagger$ Nanophotonics and Metrology Laboratory, Swiss Federal Institute of Technology Lausanne (EPFL), 1015 Lausanne, Switzerland

$\ddagger$ Bionanophotonic Systems Laboratory, Swiss Federal Institute of Technology Lausanne (EPFL), 1015 Lausanne, Switzerland

\Current affiliation: Dept. of Applied Physics and Institute for Photonic Integration, Eindhoven University of Technology, 5600 MB Eindhoven, The Netherlands $\S$ Current affiliation: Optotune, Bernstrasse 388, Switzerland

E-mail: olivier.martin@epfl.ch 
Table SI 1: Sensitivity values for a single nanoantenna computed from Fig. 3(a)-(c)

\begin{tabular}{|l|l|l|}
\hline Structure & Feature no. & $\mathbf{S}=\Delta \lambda / \Delta n \mathbf{n m} / \mathbf{R I U}$ \\
\hline Al disk & 1 & 1134 \\
\hline \multirow{4}{*}{ Si cylinder } & 1 & 9.9 \\
& 2 & 99.2 \\
\hline & 3 & 61.8 \\
& 4 & 101.3 \\
\hline \multirow{4}{*}{ Coupled nanoantenna } & 3 & 19.8 \\
& 2 & 91.4 \\
& 4 & 88.9 \\
& 5 & 61.8 \\
\hline
\end{tabular}
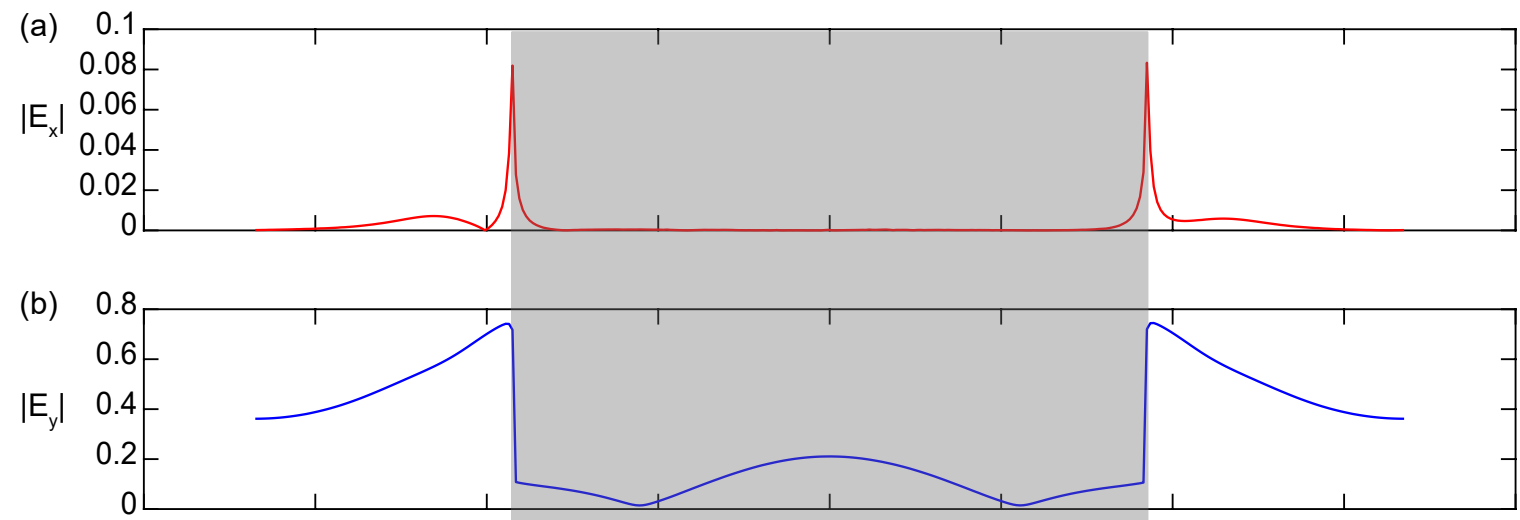

(c)

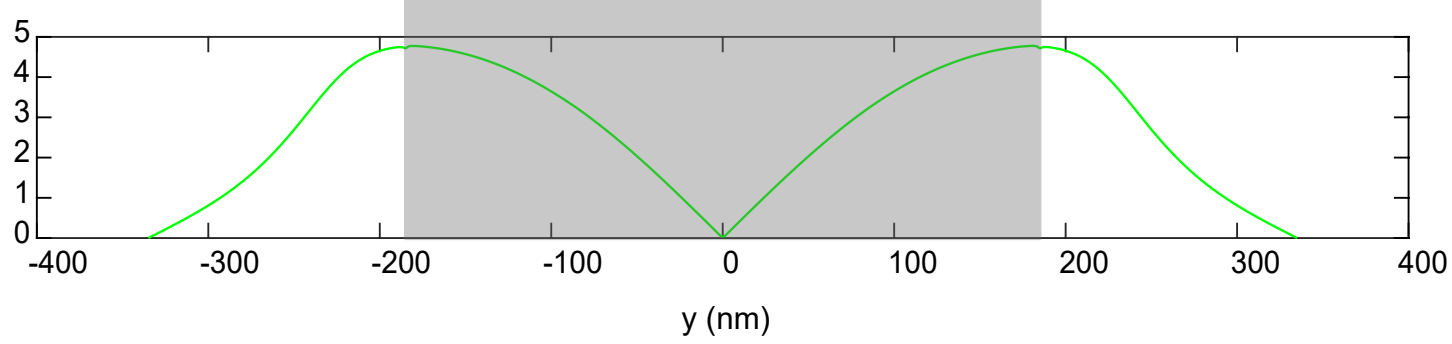

Figure SI 1: Distribution of $|\mathrm{E}|$ field: (a) $\left|\mathrm{E}_{\mathrm{x}}\right|$ (b) $\left|\mathrm{E}_{\mathrm{y}}\right|$ and (c) $\left|\mathrm{E}_{\mathrm{z}}\right|$ for the nanoantenna with undercut shown in Figure $5 \mathrm{~b}$, at $z=256 \mathrm{~nm}$ and across $y$. The shaded region shows the region inside the dielectric spacer. 